\title{
Solving Scattering from Conducting Body Coated by Thin-Layer Material by Hybrid Shell Vector Element with Boundary Integral Method
}

\author{
Lin Lei, Jun Hu, and Hao-Quan Hu \\ School of Electronic Engineering, University of Electronic Science and Technology of China, Chengdu 611731, China \\ Correspondence should be addressed to Jun Hu, hujun@uestc.edu.cn
}

Received 25 October 2011; Accepted 3 February 2012

Academic Editor: Ahmed A. Kishk

Copyright (C) 2012 Lin Lei et al. This is an open access article distributed under the Creative Commons Attribution License, which permits unrestricted use, distribution, and reproduction in any medium, provided the original work is properly cited.

\begin{abstract}
The finite element boundary integral (FEM-BI) method is widely used in the scattering and radiating problems. But for the conducting body coated by thin-layer material, plenty of fine meshes are required to discretize the geometry in the traditional FEM. It requires very expensive storage and CPU time. In this paper, the hybrid shell vector element with the boundary integral method is used to expedite the solution of thin coating problems. The shell vector elements are used to discretize thin-layer material instead of traditional tetrahedral elements. Consequently, the volume integral can be simplified into surface integral. This method reduces the number of unknowns greatly and is also extended into the complicated case of multi-thin-layer coating materials. Several numerical results are presented to prove the accuracy and efficiency of this present method.
\end{abstract}

\section{Introduction}

The electromagnetic analysis of the conducting body coated by thin-layer material has received much attention because these composite dielectric and conductor structures are used in many applications, such as the evaluation of echo from stealth aircraft and microwave integrated circuits design. Plenty of research work has been done on the efficient solution of scattering from conducting body coated by thin-layer material.

For the analysis of conductor structures coated by thinlayer material, there are many methods available. Integral equation methods based on thin dielectric sheet approximation (TDS) [1-7] avoid the volumetric discretization of material region, the computational region is only limited as the surface of conductor. A multilevel-TDS extension is also proposed for solution of conducting body coated by multithin-layer materials [8-10]. For lossy thin-layer coating, the impedance boundary condition (IBC) is also developed to simplify the electromagnetic analysis by building the relation between the equivalent surface magnetic current and equivalent surface electric current on the surface of conductor [11]. The application of the IBC into the conducting bodies of revolution coated with thin magnetic materials is also developed successfully [12]. A rigorous moment method solution of composite bodies with thin features can achieve accurate results for thin coating problems if the near singularity for near-self term integral can be tackled carefully, as shown in $[13,14]$.

Besides the integral equation methods, the finite element method (FEM) is also widely used for analysis of composite conducting body and dielectric because of its powerful ability of modeling complex inhomogeneous materials [15]. As well known, the absorption boundary condition $(\mathrm{ABC})$ is required in FEM in order to truncate the computational region. In order to combine together the advantage of FEM and integral equation, the hybrid FEM with the boundary integral method (FEM-BI) is proposed [15-23]. No ABC is needed in the FEM-BI, and the number of unknowns is reduced greatly.

Though FEM-BI has a good computational property for composite conducting body and dielectric, it is deficient for the analysis of conductor structures coated by thin-layer material. This is because plenty of fine meshes will be required for modeling thin layers if using traditional elements like tetrahedral elements. To further reduce the total number of unknowns, prism elements are used in FEM when solving 


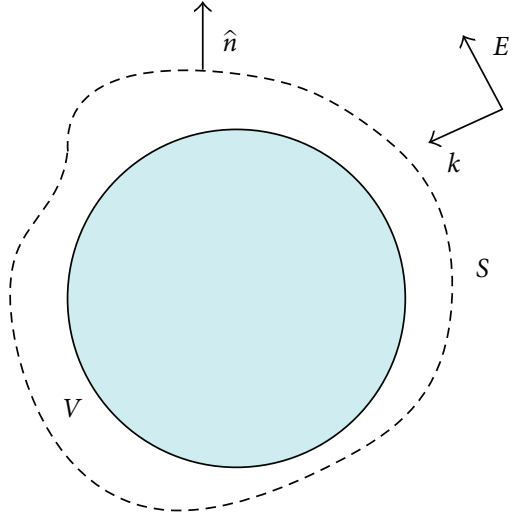

FIGURE 1: The 3D electromagnetic scattering problem by the FEMBI.

thin-coating structures [23]. In order to further enhance the computational efficiency, shell vector elements are developed in FEM, instead of prism elements [24, 25]. The volume integral can be simplified into surface integral by using shell vector elements.

In this paper, we combine the shell vector element method with the boundary integral method in order to achieve faster solution of scattering from conducting body coated by thin-layer material. The present method does not only reduce the number of unknowns but is also extended into more complicated cases of multi-thin-layer coating. This paper is organized as follows. The basic principle of the traditional FEM-BI method is reviewed in section II. Next, we show the characteristics of the shell elements, and the hybrid shell vector element method with the boundary integral method is implemented. Finally, several examples are shown to demonstrate the efficiency and accuracy of this present method. The conclusions are also given.

\section{Traditional FEM-BI Method}

Considering the electromagnetic scattering problem shown in Figure 1, the structure is illuminated by an incident plane wave $E^{\text {inc }}$ or $H^{\text {inc }}$, the $\hat{n}$ is the unit normal vector on surface $S$ and $S$ is the surface enclosing volume $V$.

The $E$ field inside volume $V$ satisfies the following equation:

$$
\nabla \times\left(\frac{1}{\mu_{r}} \nabla \times E\right)-k_{0}^{2} \varepsilon_{r} E=0,
$$

where $\mu_{r}$ and $\varepsilon_{r}$ denote the relative permeability and permittivity of the media, respectively, and $k_{0}$ is the wave number in free space.

The boundary conditions on surface $S$ are written as

$$
\begin{gathered}
\hat{n} \times\left. E\right|_{S^{-}}=\hat{n} \times\left. E\right|_{S^{+}}, \\
\hat{n} \times\left.\left(\frac{1}{\mu_{r}} \nabla \times E\right)\right|_{S^{-}}=-j k_{0} \hat{n} \times\left. H_{0}\right|_{S^{+}},
\end{gathered}
$$

where $H_{0}=\eta_{0} H$ and $\eta_{0}$ is the wave impedance in free space.
The FEM matrix can be constructed based on the variational method; the functional $F(E)$ is expressed as follows:

$$
\begin{aligned}
F(E)= & \frac{1}{2} \iiint_{V}\left[\frac{1}{\mu_{r}}(\nabla \times E) \cdot(\nabla \times E)-k_{0}^{2} \varepsilon_{r} E \cdot E\right] d V \\
& +j k_{0} \eta_{0} \iint_{S} \hat{n} \cdot\left(E \times H_{s}\right) d S .
\end{aligned}
$$

The tetrahedral element is most widely applied in traditional FEM and the electric field $E$ can be expanded by the tetrahedral element as

$$
E=\sum_{j=1}^{6} E_{j}^{e} N_{j}^{e},
$$

where $E_{j}^{e}$ and $N_{j}^{e}$ are the unknown coefficient and the basis function of the $j$ th edge, respectively.

For the surface integral term in (3), the magnetic field on the surface can be expanded by three edges of planar triangle on surface $S$ as

$$
H_{s}=\sum_{j=1}^{3} H_{j}^{s} N_{j}^{s},
$$

where $H_{j}^{s}$ and $N_{j}^{s}$ are the unknown coefficient and the basis function of the $j$ th edge, respectively.

Finally, the following FEM matrix equation is yielded from $(3)$ :

$$
[K]\{E\}+[B]\{H\}=\{0\} .
$$

In the FEM-BI method, surface integral equation method is applied on the surface $S$. The electric field integral equation (EFIE) and the magnetic field integral equation (MFIE) are used as follows:

$$
\begin{aligned}
E^{\text {inc }} & =L(\bar{J})-K(M), \\
\bar{H}^{\text {inc }} & =K(\bar{J})+L(M) .
\end{aligned}
$$

The equivalent surface electric and magnetic currents are defined as

$$
\begin{aligned}
J & =\hat{n} \times H, \\
M & =E \times \hat{n}, \\
\bar{J} & =\hat{n} \times \bar{H} .
\end{aligned}
$$

here $\bar{H}^{\text {inc }}=\eta_{0} H^{\text {inc }}$. The integral operators $L$ and $K$ are expressed, respectively, as

$$
\begin{gathered}
L(X)=j k_{0} \int_{s^{\prime}}\left[X\left(r^{\prime}\right)+\frac{1}{k_{0}^{2}} \nabla \nabla^{\prime} \cdot X\left(r^{\prime}\right)\right] G\left(r, r^{\prime}\right) d s^{\prime}, \\
K(X)=\operatorname{TY}(r)+\int_{s^{\prime}} X\left(r^{\prime}\right) \times \nabla G\left(r, r^{\prime}\right) d s^{\prime} .
\end{gathered}
$$

In (10), $Y(r)=X(r) \times \hat{n}, T=1-\Omega / 4 \pi$. For the smooth surface, $\Omega=2 \pi$. $G=e^{-j k_{0}\left|r-r^{\prime}\right|} / 4 \pi\left|r-r^{\prime}\right|$ is Green's function 
in free space. The integral terms with bars in (10) denote the principal value integrals. The singularity is excluded.

By choosing $S_{i}^{s}=\hat{n} \times N_{i}$ as the weighting function, the TE formulation can be derived as

$$
\left[P^{\mathrm{TE}}\right]\left\{E_{S}\right\}+\left[Q^{\mathrm{TE}}\right]\left\{H_{S}\right\}=\left\{b^{\mathrm{TE}}\right\},
$$

where

$$
\begin{aligned}
P_{i j}^{\mathrm{TE}} & =-\int_{S} S_{i}^{s} \cdot K\left(N_{j} \times \hat{n}\right) d s, \\
Q_{i j}^{\mathrm{TE}} & =\int_{S} S_{i}^{s} \cdot L\left(\eta_{0} \hat{n} \times N_{j}\right) d s, \\
b_{i}^{\mathrm{TE}} & =\int_{S} S_{i}^{s} \cdot E^{i} d s .
\end{aligned}
$$

Similarly, the NE formulation can be derived by choosing $\hat{n} \times S_{i}^{s}$ as the weighting function as

$$
\left[P^{\mathrm{NE}}\right]\left\{E_{S}\right\}+\left[Q^{\mathrm{NE}}\right]\left\{H_{S}\right\}=\left\{b^{\mathrm{NE}}\right\} .
$$

The TH formulation can be derived by choosing $S_{i}^{s}$ as the weighting function as:

$$
\left[P^{\mathrm{TH}}\right]\left\{E_{S}\right\}+\left[Q^{\mathrm{TH}}\right]\left\{H_{S}\right\}=\left\{b^{\mathrm{TH}}\right\} .
$$

The NH formulation can be derived by choosing $\hat{n} \times S_{i}^{s}$ as the weighting function as:

$$
\left[P^{\mathrm{NH}}\right]\left\{E_{S}\right\}+\left[Q^{\mathrm{NH}}\right]\left\{H_{S}\right\}=\left\{b^{\mathrm{NH}}\right\} .
$$

To avoid the interior resonance that occurred in some cases, the combined field integral equation (CFIE), which combines the EFIE and MFIE, can be used,

$$
[P]\left\{E_{S}\right\}+[Q]\left\{H_{S}\right\}=\{b\},
$$

where

$$
\begin{aligned}
& {[P]=\alpha\left[P^{\mathrm{TE}}\right]+\beta\left[P^{\mathrm{NE}}\right]+\gamma\left[P^{\mathrm{NH}}\right],} \\
& {[Q]=\alpha\left[Q^{\mathrm{TE}}\right]+\beta\left[Q^{\mathrm{NE}}\right]+\gamma\left[Q^{\mathrm{NH}}\right],} \\
& \{b\}=\alpha\left\{b^{\mathrm{TE}}\right\}+\beta\left\{b^{\mathrm{NE}}\right\}+\gamma\left\{b^{\mathrm{NH}}\right\} .
\end{aligned}
$$

The parameters $\alpha, \beta$, and $\gamma$ satisfy: $\alpha+\beta+\gamma=1$.

By combining (6) and (16), we get the matrix equation of the FEM-BI method as follows:

$$
\left[\begin{array}{ccc}
K_{I I} & K_{I S} & 0 \\
K_{S I} & K_{S S} & B \\
0 & P & Q
\end{array}\right]\left\{\begin{array}{c}
E_{I} \\
E_{S} \\
\bar{H}_{S}
\end{array}\right\}=\left\{\begin{array}{l}
0 \\
0 \\
b
\end{array}\right\},
$$

where $E_{I}, E_{S}$, and $\bar{H}_{S}$ are the coefficient of the electric field inside volume $V$, on surface $S$, and the magnetic field on surface $S$, respectively. (a) The prism element

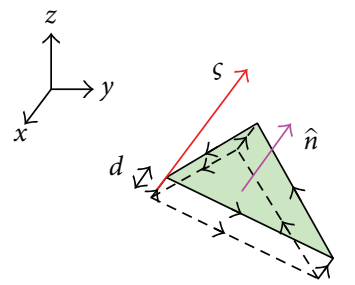

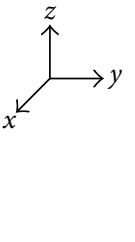

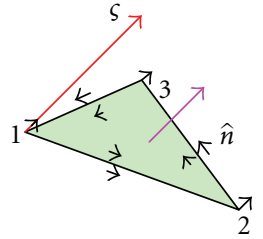

(b) The shell element
Figure 2: The structure of the prism element and the shell element.

\section{Hybrid Shell Element with Boundary Integral (Hybrid Shell Element-BI)}

The shell element is the degenerated prism element. As shown in Figure 2, there are six edge vectors along the corresponding edges in the upper triangle and bottom triangle and three normal vectors. A linear function $\beta(\varsigma)$ was used to describe the variation of the field along the normal direction, and $\nabla \beta=-\hat{n} / d$. The $\beta N_{j}(j=1,2,3)$ and $\beta^{\prime} N_{j}^{\prime}(j=1,2,3)$ are the edge basis functions in the upper and bottom triangle, respectively. The $L_{j}, j=1,2,3$ is the normal basis function at node $j$.

By the shell element, the electric field is expanded as follows:

$$
E^{e}=\sum_{j=1}^{3}\left(E_{j}^{e} \beta N_{j}+E_{j}^{\prime e} \beta^{\prime} N_{j}^{\prime}\right)+\sum_{j=1}^{3} E_{n j}^{e} L_{j}^{e} \hat{n},
$$

where $\beta^{\prime}=1-\beta, N_{j}=\left(L_{j 1}^{e} \nabla L_{j 2}^{e}-L_{j 2}^{e} \nabla L_{j 1}^{e}\right) l_{j}^{e}, N_{j}^{\prime}=$ $\left(L_{j 1}^{e} \nabla L_{j 2}^{e}-L_{j 2}^{e} \nabla L_{j 1}^{e}\right) l_{j}^{e}, E_{j}^{e}$ is the expansion coefficient of the $j$ th edge vector in the upper triangle, $E_{j}^{\prime}$ is the expansion coefficient of the $j$ th edge vector in the bottom triangle, $E_{n j}^{e}$ is the expansion coefficient of the normal vector at node $j$.

Based on (3) and (19), we attain

$$
\begin{aligned}
\iint_{v}\{ & \nabla \times\left[\beta N_{i}^{e}+\beta^{\prime} N_{i}^{e}+L_{i}^{e} \hat{n}\right] \cdot \nabla \\
& \times \frac{\left[\sum_{j=1}^{3}\left(E_{j}^{e} \beta N_{j}^{e}+E_{j}^{\prime e} \beta^{\prime} N_{j}^{e}\right)+\sum_{j=1}^{3} E_{n j}^{e} L_{j}^{e} \hat{n}\right]}{\mu_{r}} \\
& -k_{0}^{2} \varepsilon_{r}\left[\beta N_{i}^{e}+\beta^{\prime} N_{i}^{e}+L_{i}^{e} \hat{n}\right] \\
& \left.\cdot\left[\sum_{j=1}^{3}\left(E_{j}^{e} \beta N_{j}^{e}+E^{\prime e} \beta^{\prime} N_{j}^{e}\right)+\sum_{j=1}^{3} E_{n j}^{e} L_{j}^{e} \hat{n}\right]\right\} d V \\
= & j k_{0} \eta_{0} \sum_{j=1}^{3} H_{j}^{s} \iint_{s} N_{i}^{s} \cdot\left(\hat{n} \times N_{j}^{s}\right) d s .
\end{aligned}
$$


For the shell element model, the volume integral can be simplified as:

$$
\begin{gathered}
\iint_{V}\left(\nabla \times \beta N_{i}^{e}\right) \cdot\left(\nabla \times \beta N_{j}^{e}\right) d v \\
=\int_{-1 / 2}^{1 / 2} \beta \beta d \varsigma \iint_{s}\left(\nabla \times N_{i}^{e}\right) \cdot\left(\nabla \times N_{j}^{e}\right) d s \\
\quad-\int_{-1 / 2}^{1 / 2} \frac{\beta}{d} d \varsigma \iint_{s}\left(\nabla \times N_{i}^{e}\right) \cdot S_{j}^{e} d s \\
\quad-\int_{-1 / 2}^{1 / 2} \frac{\beta}{d} d \varsigma \iint_{s} S_{i}^{e}\left(\nabla \times N_{j}^{e}\right) d s+\int_{-1 / 2}^{1 / 2} \frac{1}{d^{2}} d s \iint_{s} S_{i}^{e} \cdot S_{j}^{e} d s \\
\iint_{V}\left(\nabla \times L_{i}^{e} \hat{n}\right) \cdot\left(\nabla \times \beta^{\prime} N_{j}^{e}\right) d v \\
=\int_{-1 / 2}^{1 / 2} \beta^{\prime} d \varsigma \iint_{s}\left(\nabla L_{i}^{e} \times \hat{n}\right) \cdot\left(\nabla \times N_{j}^{e}\right) d s \\
\quad+\int_{-1 / 2}^{1 / 2} \frac{1}{d} d \varsigma \int_{s}\left(\nabla L_{i}^{e} \times \hat{n}\right) \cdot S_{j}^{e} d s,
\end{gathered}
$$

where $S_{i}^{e}=\hat{n} \times N_{i}^{e}, S_{j}^{e}=\hat{n} \times N_{j}^{e}$ and $d$ is the thickness of the thin-layer material.

Obviously, the integration along the normal direction can be calculated analytically. So the volume integral can be simplified into the surface integral.

For the conducting object coated by thin-layer material, the electric field in the bottom surface of shell element must be zero, only the integral in the upper surface is needed.

The hybrid shell element-BI can also be extended into the conducting body coated by multi-thin-layered material. For example, for the conducting body coated by three-thin-layer material, the matrix equation is expressed as:

$$
\left[\begin{array}{ccccccc}
K_{n n 1} & K_{n u 1} & & & & & 0 \\
K_{u n 1} & K_{u u 1} & K_{b n 2} & K_{b u 2} & & & \\
& K_{n b 2} & K_{n n 2} & K_{n u 2} & & \\
& K_{u b 2} & K_{u n 2} & K_{u u 2} & K_{b n 3} & K_{b u 3} & \\
& & & K_{n b 3} & K_{n n 3} & K_{n u 3} & \\
& & & K_{u b 3} & K_{u n 3} & K_{u u 3} & B \\
0 & & & & & P & Q
\end{array}\right]\left\{\begin{array}{c}
E_{n 1} \\
E_{u 1} \\
E_{n 2} \\
E_{u 2} \\
E_{n 3} \\
E_{u 3} \\
H_{s}
\end{array}\right\}=\left\{\begin{array}{l}
0 \\
0 \\
0 \\
0 \\
0 \\
0 \\
b
\end{array}\right\},
$$

where $E_{n 1}$ and $E_{u 1}$ are the expansion coefficients of the electric field in the normal and upper surface of the first layer, respectively; $E_{n 2}$ and $E_{u 2}$ are the expansion coefficients of the electric field in the normal and upper surface of the second layer, respectively, $E_{n 3}$ and $E_{u 3}$ are the expansion coefficients of the electric field in the normal and upper surface of the third layer, respectively.

\section{Numerical Results}

To demonstrate the accuracy and efficiency of the present method, some typical numerical results are shown here.

The first example is a conducting sphere coated by magnetic material, shown in Figure 3. The frequency is $300 \mathrm{MHz}$. The radius of the conducting sphere is $0.3367 \mathrm{~m}$, and the

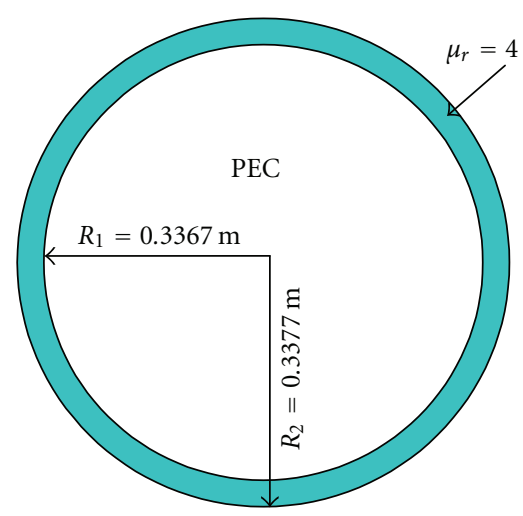

FIgure 3: Conducting sphere coated by magnetic material.

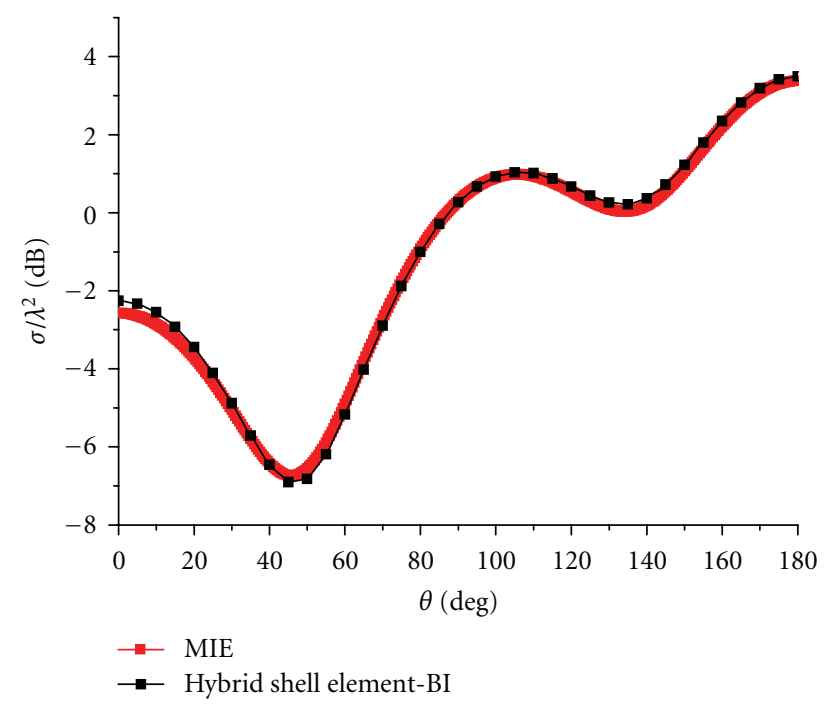

FIgURE 4: The bistatic RCS of the conducting sphere coated by magnetic material by the hybrid shell element-BI.

thickness of the coating layer is $0.001 \mathrm{~m}$. The relative permeability is $\mu_{r}=4$ and the relative permittivity is $\varepsilon_{r}=1$. In the hybrid shell element with BI, the mesh density of 15 elements per wavelength is enough for achieving reasonable accuracy, but in traditional FEM, at least 32 elements per wavelength are required in order to mesh this very thin layer successfully. Table 1 demonstrates the comparisons between the two methods. As shown in Figure 4, the results by the hybrid shell element with BI agree with the ones of MIE method very well. And, the number of the unknowns by the traditional FEM-BI and the present method is 15167 and 2249, respectively. Obviously, the advantage of the present method over the traditional FEM-BI in reducing the number of unknowns is very remarkable.

To further investigate the accuracy of the hybrid shell element-BI, Figure 6 shows the bistatic RCS of the conducting sphere coated by thin dielectric material, the geometry is shown in Figure 5, $k_{0} a=6.28$, the thickness of the thin layer is $a / 100$, and the relative permittivity is $\varepsilon_{r}=4$, where $k_{0}$ is the wave number in free space and $a$ is the radius of the conducting sphere. The mesh density used here is only 
TABLE 1: The comparison of the FEM-BI and the hybrid shell element-BI. Bistatic RCS of the conducting sphere coated by magnetic material is calculated.

\begin{tabular}{lccccc}
\hline Method & Mesh density (wavelength) & Node number & Element type & Element number & Unknowns \\
\hline FEM-BI & 32 elements & 3033 & Tetrahedral elements & 9132 & 15167 \\
Hybrid shell element-BI & 15 elements & 323 & Shell elements & 642 & 2249 \\
\hline
\end{tabular}

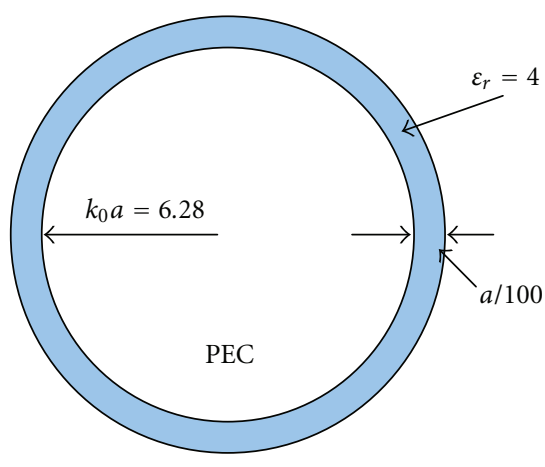

FIGURE 5: Coated metal sphere.

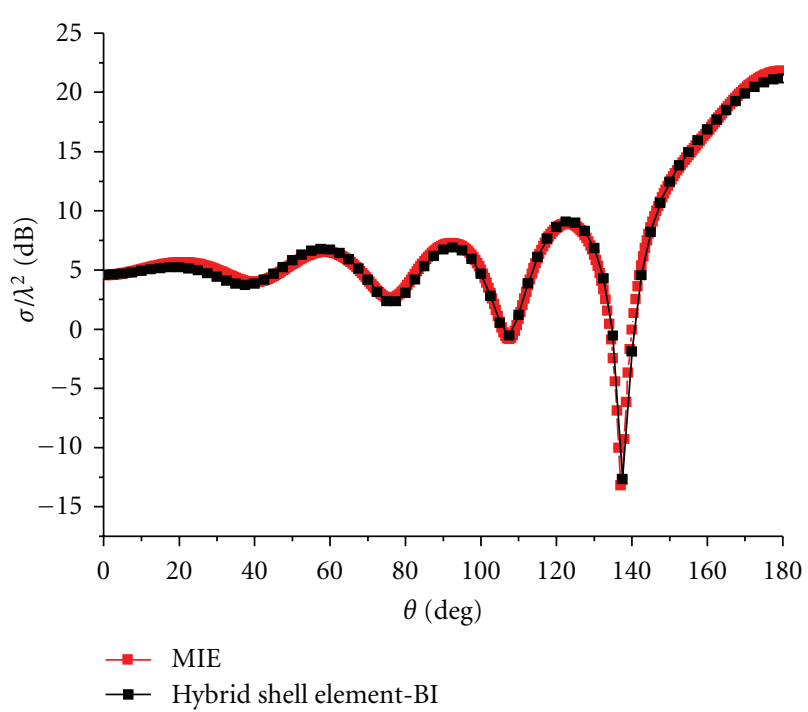

FIGURE 6: The bistatic RCS of the conducting sphere coated by thin dielectric material by the hybrid shell element with BI.

6 elements per wavelength. As shown in Figure 6, the results by the hybrid shell element -BI agree well with the ones of the MIE method. In this case, the number of unknowns is 10530.

Figure 8 shows the bistatic RCS of the conducting cube coated by thin material in horizontal polarization. The geometry is shown in Figure 7. The length of the cube is $0.1 \mathrm{~m}$, and the thickness of the coated layer is $0.001 \mathrm{~m}$. The wavelength is $0.1 \mathrm{~m}$. The incident angle of the plane wave is $\theta^{\text {inc }}=45^{\circ}, \varphi^{\text {inc }}=0^{\circ}$.

From Figure 8, it is shown clearly that the results by hybrid shell element-BI agree well with the ones by the commercial software FEKO. The number of unknowns in the hybrid shell element-BI is 5630, but the one in the traditional FEM-BI is 7760 .

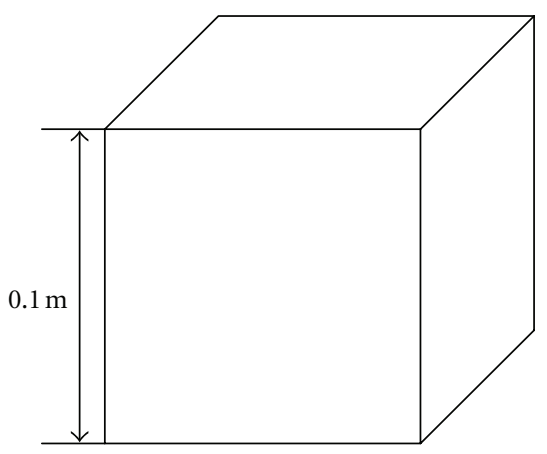

FIGURE 7: Conducting cube coated by thin material.

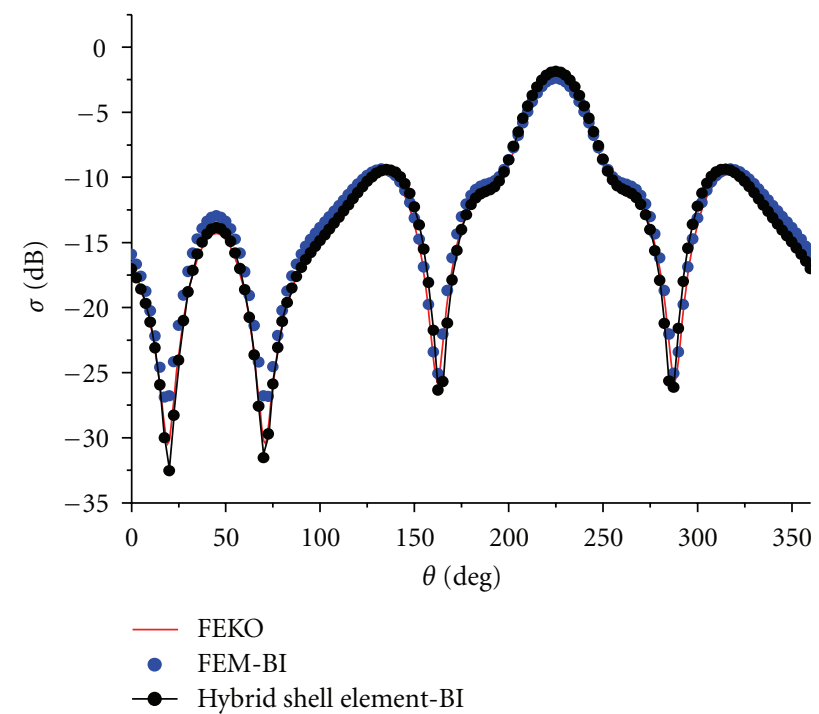

FIgURE 8: The bistatic RCS of the coated metallic cube.

The solving CPU time by the present method is only onethird of the one by the traditional FEM-BI. The details are summarized in Table 2. Obviously, compared with the FEM$\mathrm{BI}$, the hybrid shell element with BI can save memory and CPU time greatly.

Figure 9 shows a conducting sphere with radius of $0.5 \lambda_{0}$ coated by three-thin-layer material, the thickness of each layer is $0.01 \lambda_{0}, \mu_{r 1}=1.0, \varepsilon_{r 1}=2.0, \mu_{r 2}=2.0-j 2.0$, $\varepsilon_{r 2}=2.0-j 2.0, \mu_{r 3}=2.0$, and $\varepsilon_{r 3}=2.0$. The number of unknowns is 9591 and the total memory required is $736 \mathrm{Mb}$, but the number of unknowns in the traditional FEM-BI is 18775 and the total memory required is $2.9 \mathrm{~Gb}$. The bistatic RCS computed by the hybrid shell element-BI is shown in Figure 10, the one by the MIE method is also shown for comparison. The lines marked with dot and square denote 
TABLE 2: The comparison of the FEM-BI and the hybrid shell element-BI. Bistatic RCS of the conducting cube coated by thin material (mesh density with 10 elements/ $\lambda$ ).

\begin{tabular}{lcccc}
\hline Method & Node number & Memory & Element number & Unknowns \\
\hline FEM-BI & 1474 & $480 \mathrm{Mb}$ & 4404 & 7760 \\
Hybrid shell element-BI & 806 & $250 \mathrm{Mb}$ & 1608 & 5630 \\
\hline
\end{tabular}

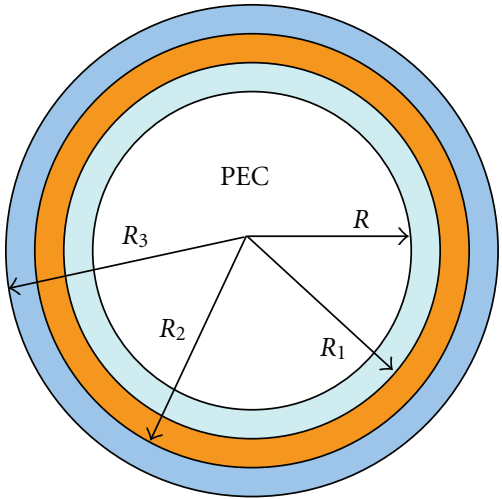

FIgURE 9: Conducting sphere coated by three-thin-layer materials.

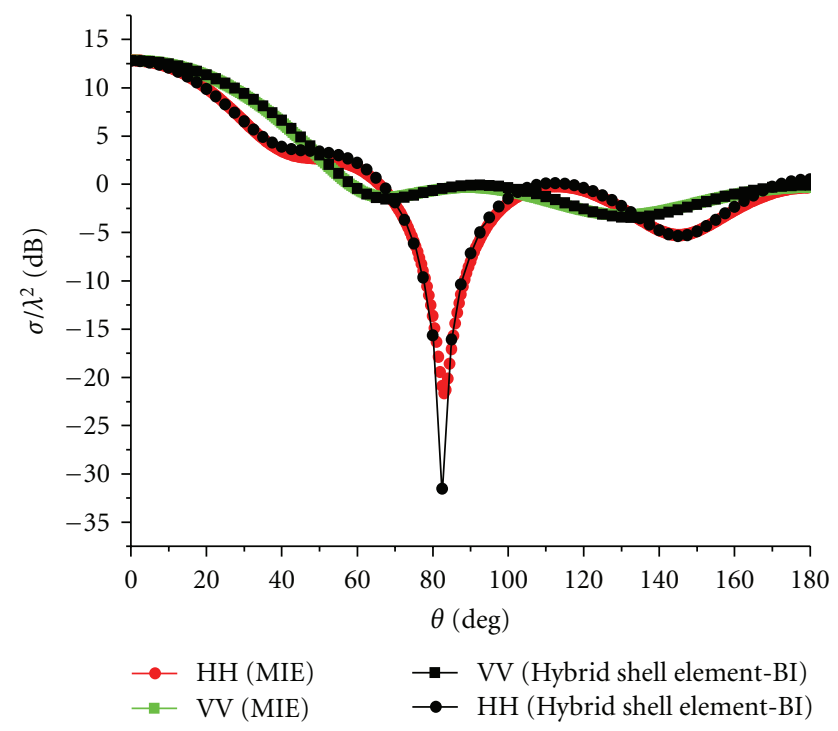

Figure 10: The bistatic RCS of the conducting sphere coated by three-thin-layer material.

the curves of results in $\mathrm{HH}$ polarization and VV polarization, respectively. Obviously, they agree well with each other.

\section{Conclusions}

In this paper, the hybrid shell element with $\mathrm{BI}$ is developed to achieve efficient solution of scattering from the conducting body coated by thin-layer electric or magnetic material. Compared with the traditional FEM-BI, the present method reduces the number of unknowns greatly. Numerical results show that the hybrid shell element with BI is accurate and effective in solving the scattering problems of the conducting body coated with thin layer, also valid for the case of multithin-layer material coating.

\section{Acknowledgment}

The work is supported by the Nature Science Foundation of China (no. 60971032).

\section{References}

[1] R. F. Harrington and J. R. Mautz, "An impedance sheet approximation for thin dielectric shells," IEEE Transactions on Antennas and Propagation, vol. 23, no. 4, pp. 531-534, 1975.

[2] C. C. Lu, "A modified thin dielectric approximation for calculation of em scattering by dielectric objects with thin material coating," in Proceedings of the IEEE Antennas and Propagation Society International Symposium, pp. 2809-2812, June 2007.

[3] I. T. Chiang and W. C. Chew, "Thin dielectric sheet simulation by surface integral equation using modified RWG and pulse bases," IEEE Transactions on Antennas and Propagation, vol. 54, no. 7, pp. 1927-1934, 2006.

[4] I. T. Chiang and W. C. Chew, "A coupled PEC-TDS surface integral equation approach for electromagnetic scattering and radiation from composite metallic and thin dielectric objects," IEEE Transactions on Antennas and Propagation, vol. 54, no. 11, pp. 3511-3516, 2006.

[5] C. P. Davis and W. C. Chew, "An alternative to impedance boundary conditions for dielectric-coated PEC surfaces," in Proceedings of the IEEE Antennas and Propagation Society International Symposium, pp. 2785-2788, June 2007.

[6] G. Pan and R. M. Narayanan, "Electromagnetic scattering from a dielectric sheet using the method of moments with approximate boundary condition," Electromagnetics, vol. 24, no. 5, pp. 369-384, 2004.

[7] S. He, Z. Nie, and J. Hu, "Numerical solution of scattering from thin dielectric-coated conductors based on TDS approximation and em boundary conditions," Progress in Electromagnetics Research, vol. 93, pp. 339-354, 2009.

[8] S. He, Z. Nie, S. Yan, and J. Hu, "Multi-layer TDS approximation used to numerical solution for dielectric objects," in Proceedings of the Asia Pacific Microwave Conference (APMC '08), 2008.

[9] S. He, Z. Nie, J. Wei, and J. Hu, "Numerical solution for dielectric-coated PEC targets based on multi-layer TDS approximation," in Proceedings of the Asia Pacific Microwave Conference (APMC'08), December 2008.

[10] S. He, Z. Nie, J. Wei, and J. Hu, "A highly efficient numerical solution for dielectric-coated PEC targets," Waves in Random and Complex Media, vol. 19, no. 1, pp. 65-79, 2009.

[11] D. S. Wang, "Limits and validity of the impedance boundary condition on penetrable surfaces," IEEE Transactions on Antennas and Propagation, vol. 35, no. 4, pp. 453-457, 1987. 
[12] A. A. Kishk and R. K. Gordon, "Electromagnetic scattering from conducting bodies of revolution coated with thin magnetic materials," IEEE Transactions on Magnetic, vol. 30, no. 5, pp. 3151-3155, 1994.

[13] P. M. Goggans, A. A. Kishk, and A. W. Glisson, "A systematic treatment of conducting and dielectric bodies with arbitrarily thick or thin features using the method of moments," IEEE Transactions on Antennas and Propagation, vol. 40, no. 5, pp. 555-559, 1992.

[14] P. M. Goggans, A. A. Kishk, and A. W. Glisson, "The use of "Near-Self" impedance elements in the MM solution for scattering from composite bodies with thin features," in Proceedings of the IEEE Antennas and Propagation Society International Symposium Digest, pp. 1484-1487, June 1991.

[15] J.-M. Jin, The Finite Element Method in Electromagnetics, John Wiley \& Sons, New York, NY, USA, 1993.

[16] A. J. Sangster and H. Wang, "A combined FEM/MoM technique of coupling radiating apertures in rectangular waveguide," IEEE Transactions on Magnetics, vol. 31, no. 3, pp. 15541557, 1995.

[17] M. W. Ali, T. H. Hubing, and J. L. Drewniak, "A hybrid FEM/MOM technique for electromagnetic scattering and radiation from dielectric objects with attached wires," IEEE Transactions on Electromagnetic Compatibility, vol. 39, no. 4, pp. 304-314, 1997.

[18] J. L. Volakis, T. Özdemir, and J. Gong, "Hybrid finite-element methodologies for antennas and scattering," IEEE Transactions on Antennas and Propagation, vol. 45, no. 3, pp. 493-507, 1997.

[19] X. Q. Sheng, J. M. Jin, J. M. Song, C. C. Lu, and W. C. Chew, "On the formulation of hybrid finite-element and boundaryintegral methods for 3-D scattering," IEEE Transactions on Antennas and Propagation, vol. 46, no. 3, pp. 303-311, 1998.

[20] J.-M. Jin and J.-L. Volakis, "A hybrid finite element method for scattering and radiation by microstrip patch antennas and arrays residing in a cavity," IEEE Transactions on Antennas and Propagation, vol. 39, no. 11, pp. 1598-1604, 1991.

[21] J.-M. Jin and J.-L. Volakis, "A finite-element-boundary integral formulation for scattering by three-dimensional cavitybacked apertures," IEEE Transactions on Antennas and Propagation, vol. 39, no. 1, pp. 97-104, 1991.

[22] Y. Ji, M. Ali, and T. H. Hubing, "EMC applications of the EMAP5 hybrid FEM/MOM code," in Proceedings of the IEEE International Symposium on Electromagnetic Compatibility, vol. 1, pp. 177-181, Denver, Colo, USA, August 1998.

[23] D. Wulf and R. Bunger, "A new hybrid finite element method for thin material structures," in Proceedings of the IEEE International Symposium on Antennas and Propagation, 2008.

[24] Z. Ren, "Degenerated whitney prism elements-general nodal and edge shell elements for field computation in thin structures," IEEE Transactions on Magnetics, vol. 34, no. 5, pp. 2547 2550, 1998.

[25] E. Abenius and F. Edelvik, "Thin sheet modeling using shell elements in the finite-element time-domain method," IEEE Transactions on Antennas and Propagation, vol. 54, no. 1, pp. 28-34, 2006. 

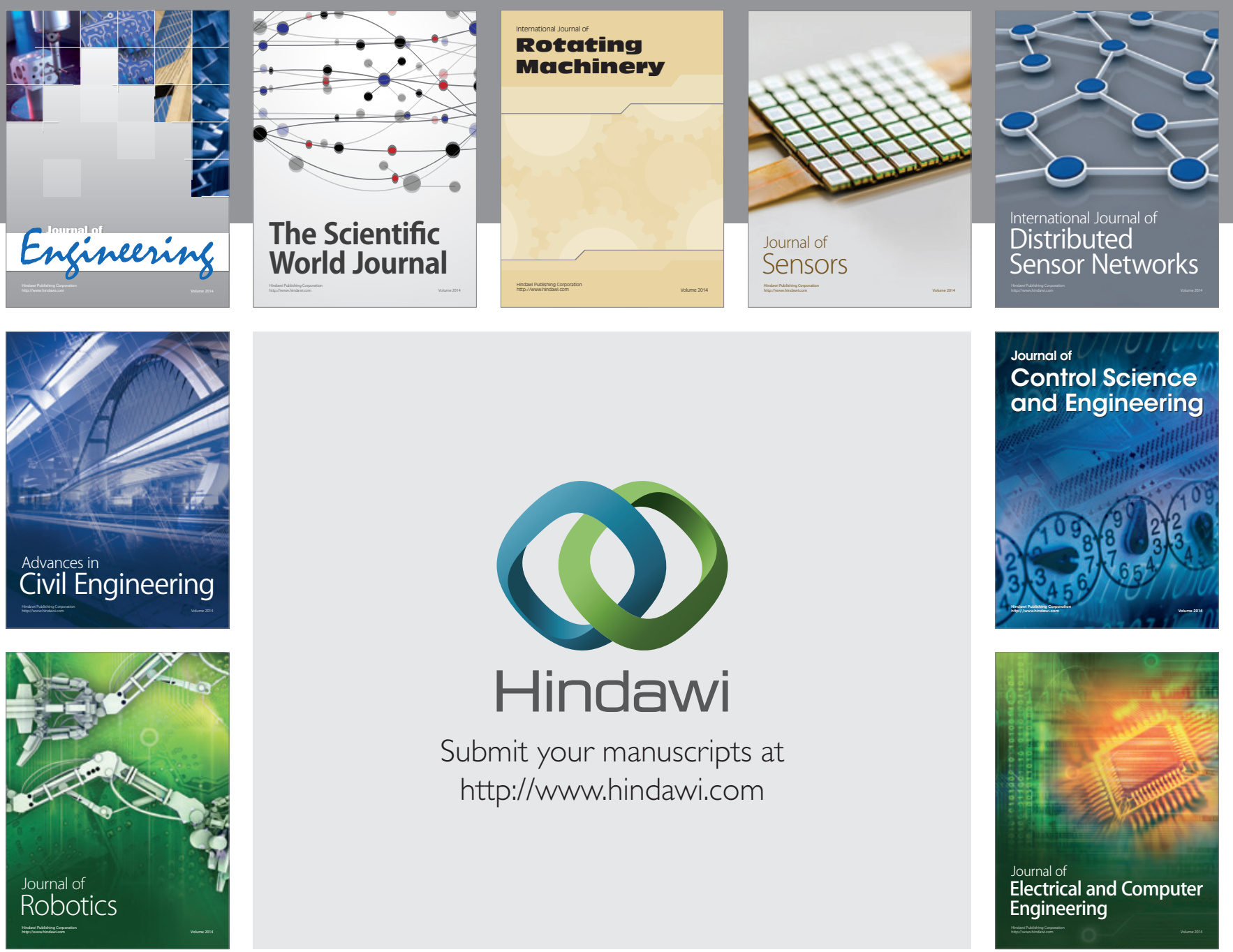

Submit your manuscripts at

http://www.hindawi.com
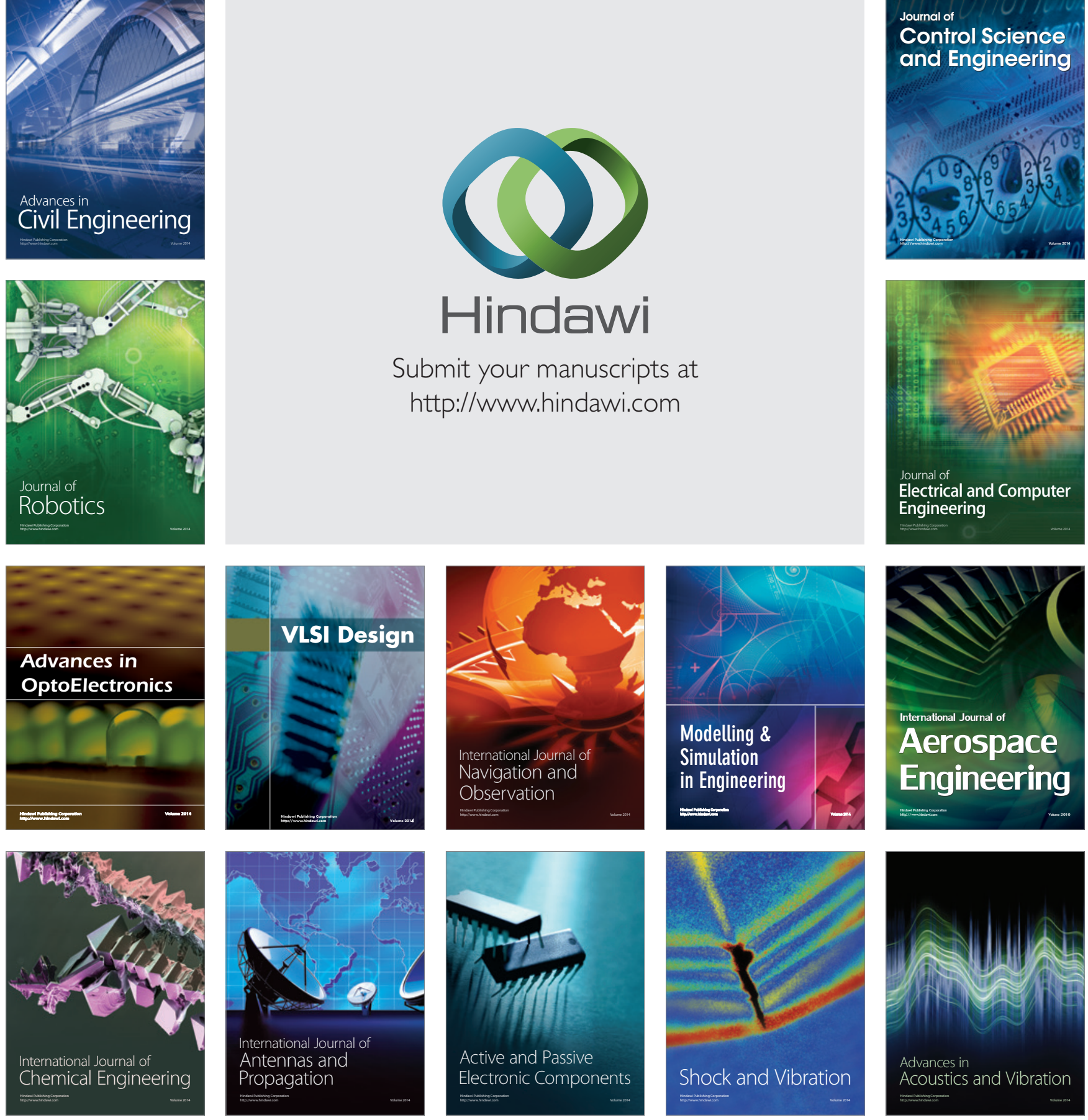